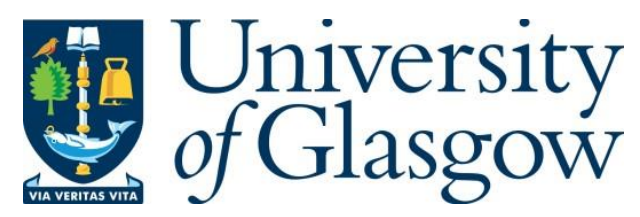

Mangano, G. R., Cipolotti, L., Turriziani, P., Giammusso, I., Smirni, D., Zhaoping, L., Bracco, M. and Oliveri, M. (2019) Exploring the neural correlates of the reversed letter effect: evidence from left and right parietal patients. Neuroscience Letters, 699, pp. 217 224. (doi:10.1016/j.neulet.2019.02.017)

There may be differences between this version and the published version. You are advised to consult the publisher's version if you wish to cite from it.

http://eprints.gla.ac.uk/180842/

Deposited on: 6 March 2019

Enlighten - Research publications by members of the University of Glasgow http://eprints.gla.ac.uk 


\section{Exploring the neural correlates of the reversed letter effect: evidence from left and right parietal patients.}

Giuseppa Renata Mangano ${ }^{1,2}$, Lisa Cipolotti $^{3}$, Patrizia Turriziani ${ }^{1,2}$, Isabella Giammusso $^{1}$, Daniela Smirni ${ }^{1,2}$, Li Zhaoping ${ }^{4}$, Martina Bracco ${ }^{5}$ and Massimiliano Oliveri $^{1,2,6}$

1. Department of Psychology, Educational Science and Human Movement, University of Palermo, Palermo, Italy

2. NeuroTeam Life and Science, Palermo, Italy

3. Department of Neuropsychology, National Hospital for Neurology and Neurosurgery, Queen Square, London, United Kingdom

4. Department of Computer Science, University College London, London, United Kingdom

5. Centre for Cognitive Neuroimaging, Institute of Neuroscience and Psychology, University of Glasgow, Glasgow, United Kingdom

6. IRCCS Santa Lucia Foundation, Rome, Italy

\section{Corresponding author:}

Giuseppa Renata Mangano, Dipartimento di Scienze Psicologiche, Pedagogiche, dell'Esercizio Fisico e della Formazione, Viale delle Scienze, Edificio 15, 90128 Palermo, Italy. Tel/Fax: +39 09123897750, e-mail: renata.mangano@unipa.it 


\section{Abstract}

To investigate the hemispheric lateralization of attentional processes during visual search tasks depending on the stimulus material embedding the target, twelve patients with unilateral left $(n=7)$ or right $(n=5)$ parietal lesions and 20 age and education matched healthy controls $(\mathrm{HC})$ were recruited. We used a visual search task for a uniquely tilted oblique bar embedded in an object shape ' $\mathrm{N}$ ' or in its mirror reversal ' $И$ '. The accuracy and the averaged reaction times (RTs) in each stimulus type ('N' or 'U') were analysed.

$\mathrm{HC}$ presented significantly longer RTs when the target bar was embedded in ' $\mathrm{N}$ ' among its mirror reversed ' $И$ ' $(p<0.05)$. This "reversed letter effect" was also found in the right parietal patients $(p<.001)$, while no evidence of a reversed letter effect was found in the left parietal patients.

Keywords: parietal cortex; top-down attention; visual search asymmetries.

\section{Introduction}

Visual search asymmetries refer to unexpected asymmetric behaviour during visual search tasks based on an apparently symmetrical design. Such asymmetries have been described in healthy populations using a number of paradigms with letters, including cancellation [1] and visual search tasks [2-4]. The general finding of these behavioural studies is that it is harder to find a letter in familiar canonical view among mirror reversed letters than vice-versa [5]. This phenomenon is named the 'reversed letter effect' and highlights the paradoxical role that the familiarity of the target exerts in slowing down the search. The main hypothesis proposed to explain this phenomenon points to the stronger preattentive salience for the novel reversed 
target letter and/or the faster rejection of familiar letters distractors (for review see [5]).

Alternatively but not exclusively, other findings may suggest an involvement of language and reading processes. Cross-linguistic studies documented that the reversed letter effect is present in participants having longer experience with the alphabetical characters employed in the visual search tasks but not in those participants who are not familiar with such alphabetical characters [3-4].

A re-interpretation of the reversed letter effect has been proposed by Zhaoping and Frith [6], who explicated the paradoxical effect exerted by familiarity in terms of a clash between bottom-up and top-down processes. They asked observers to search for a uniquely oriented bar in a search display containing a familiar letter 'N' among many of its mirror reversals ' $U$ '. In this paradigm, the unique target is the low-level feature, the left tilted oblique bar in ' $N$ ', while the shape of the letter or the reversed letter is task-irrelevant. They found that RTs for gaze to arrive at the target oblique bar were the same whether this bar was in letter ' $N$ ' or its mirror reversal. On the other hand, the latency between the gaze arrival and the participant's button press to report the target was longer when the target bar was in letter ' $N$ '. They reasoned that the letter in a familiar view activates the top-down shape recognition early, while the letter in an unfamiliar view requires mental rotation before accessing letter shape recognition. This further stage postpones top-down shape recognition and thus leads to bottom up processes to emerge and guide the search.

A previous repetitive transcranial magnetic stimulation (rTMS) study employed this task to explore the neural correlates of the reversed letter effect [7]. $1 \mathrm{~Hz} r T M S$ on the left but not right posterior parietal cortex (PPC) speeded up the performance when the target was in the letter ' $N$ ' among reversed-Ns, while this speed-up was 
weaker or non-significant when the target was in the reversed-N. Interestingly, rTMS of the right PPC speeds up an analogous visual search task, involving the same interference by a task-irrelevant high-level shape on the detection of a low-level uniquely oriented bar, except that the high-level shape has no linguistic valence (i.e., a target $X$ had to be found among distractors $X$ and + ) [8] (see Figure 3 ).

These results suggest that top-down interference on target detection in visual search tasks shows hemispheric differences depending on the stimulus material embedding the target itself: i.e. that the right and left posterior parietal cortices mediate top-down processes in the visual search tasks respectively for non-linguistic or linguistic stimulus materials.

Here we hypothesized that a lesion in the brain areas involved in the top-down letter recognition could modulate the reversed letter effect, with a selective reduction of the reversed letter effect in patients with left parietal lesions as compared with right parietal ones.

\section{Material and methods}

\subsection{Participants}

Twelve patients with unilateral parietal lesions were recruited through the databases of Ospedali Riuniti Villa Sofia-Cervello (Palermo), Centro Studi e Ricerche in Neuroscienze Cognitive (Cesena) and NeuroTeam Life and Science Research Institute (Palermo). Inclusion criteria were: 1)age range 18-75 years; 2)level of education $\geq 8$ years; 3 )no history of previous psychiatric disorder or alcohol or drugs abuse; and 4)unilateral parietal lesion identified on CT or MRI scan. Seven patients with focal left parietal damage (LPpt) (age: $M=47.57, S D=13.79$; education: $M=14.57, S D=4.15$ ) and five patients with focal right parietal damage (RPpt) (age: $M=52, S D=17.1$; education: $M=12.8, S D=3.19$ ) were recruited. Tumour patients were 
tested after undergoing neurosurgery. The mean chronicity of the lesions was 29.29 months in the LPpt group ( $S D=30.96$ months) and 14.80 months in the RPpt group $\left(S D=7.26\right.$ months; $\left.M_{d}=14.64,95 \% \mathrm{Cl}:-3.42,42.26, d=.66, p=.20\right)$.(Table 1).

None of the patients showed signs of neglect as assessed by the Behavioural Inattention Test (BIT) [9]. Four LPpt showed language deficits in a clinical interview investigating spoken language, naming, comprehension and repetition. These patients underwent a formal assessment by means of the BADA [10] or the ENPA [11] batteries. Patients 1, 3 and 5 were tested on the BADA and their performance was clinically classified as global aphasia, fluent aphasia and fluent aphasia, respectively. Patient 7 was tested on the ENPA battery and his performance was clinically classified as non-fluent aphasia. For the other patients, informal testing indicated that they did not have aphasia. No reading deficits or dyscalculia were found. CT/MRI digitalized images were available for five LPpt and three RPpt and were mapped using MRlcro software [12]. The region of maximum overlap was extracted (Figure 1) and, the number of voxels corresponding to the mapped lesion was calculated for each patient. The median and the interquartile range for LPpt and RPpt, respectively, were: $2576(I Q R=3160)$ and $2990(I Q R=2006)$.

Twenty $\mathrm{HC}$ matched for age $(M=47.3, S D=17.5)$ and education $(M=14.4, S D=3.5)$ were also recruited.

All participants were Italian native speakers and had normal or corrected to normal vision. Handedness was assessed with a clinical interview asking patients for three questions (e.g., 'Which hand do you prefer to write?', 'Which foot do you prefer to kick with?', 'Which eye do you use when using only one eye?'). HC underwent to the Edinburgh Handedness Inventory (laterality quotient: $M=76, S D=17$ ) [13]. All participants were right-handed. 
Informed consent was obtained for each participant. The study was carried out in accordance with the Code of Ethics of the World Medical Association (Declaration of Helsinki).

TABLE 1

FIGURE 1

\subsection{Neuropsychological investigation}

A battery of neuropsychological tests standardized for Italian population [14] and assessing general intellectual functioning [15], word retrieval [11], phonemic verbal fluency [16] memory [16-17], visual-perceptual [9] and executive functions [18] was administered to the patients. The two groups of patients did not differ in their visual perceptual functions $(p=.93)$. The LPpt showed lower scores than the RPpt in all the other cognitive tasks. Two tailed t-tests revealed that the differences between the two groups were not significant except for the Rey's figure-b delayed recall $(p=.03)$ (Table 2).

\section{TABLE 2}

\subsection{Experimental investigation}

\subsubsection{Experiment 1. Visual search task with linguistic stimuli.}

We used a feature visual search task employed in previous HC and rTMS studies [67] (Figure 2).

\section{FIGURE 2}

The task was to search for an oblique bar uniquely tilted from vertical in one direction, clockwise or anti-clockwise, while non-target oblique bars were tilted in the opposite direction. Each oblique bar was contained in an object-shape ' $N$ ' or ' $U$ ', 
which was the mirror-reversal of ' $N$ '. We call the two stimulus types 'target-in- $N$ stimulus' and 'target-in-reversed-N stimulus', respectively.

The target was randomly presented in the left or right half of the display, in equal number of trials, on a 14-inch monitor. Display was viewed at a distance of 55 centimetres on a white background. The fixation stimulus was a black cross at the display centre. We used a set size of the search array (6x8), spanning $18.5^{\circ} \times 28.5^{\circ}$ in visual angle. Each stimulus bar was $.01^{\circ} \times 1.04^{\circ}$ in visual angle. Each item in the search display had its horizontal and vertical positions randomly jittered from the regular grid positions. The target could appear randomly and counterbalanced at any positions of the grid, except in the central 3 columns of the search array.

\subsubsection{Procedure}

All the participants performed 1 practice trial per stimulus type. The words "letter" or "N" were never mentioned. Participants were instructed to search for the target item containing a uniquely tilted oblique bar that was tilted in the opposite direction from the uniformly oriented oblique bars in the distractors. They were asked to press a left or right button as fast as possible to indicate whether the target was in the left or right half of the display, respectively. The patients pressed the buttons with the index or middle fingers of the ipsilesional hand. Since the LPpt responded with the left hand, to control for this factor the twenty $\mathrm{HC}$ were assigned in two equivalent groups according to the hand used to respond to the task: $10 \mathrm{HC}$ responding with their preferred hand (HC pref. hand) (age: $M=44.4, S D=15.7$; education: $M=14.3, S D=3.9$; laterality quotient: $M=73, S D=19) ; 10 \mathrm{HC}$ responding with their non-preferred hand ( $\mathrm{HC}$ non-pref. hand) (age: $M=50.2, S D=19.4$; education: $M=14.6, S D=3.2$; laterality quotient: $M=79, S D=15)$. Mean age $(p=.53$,$) , education (p=.79)$ and laterality quotients $(p=.47)$ did not differ between the two HC groups. 
The search was conducted with overt eye movements. Participants viewed 50 stimulus displays, with 25 trials for each stimulus type randomly interleaved.

Participants had to ignore the vertical bars and were informed that the uniquely oriented target bar could be randomly tilted clockwise or counter-clockwise from vertical in each trial. Each trial started with a fixation stimulus lasting $600 \mathrm{~ms}$, followed by blank screen lasting $200 \mathrm{~ms}$, followed by the search display, staying on the screen till the participant's button press.

Button presses and RTs were recorded using PsyScope for Mac OS X [19].

\subsubsection{Experiment 2. Visual search task with non-linguistic stimuli.}

All patients but one (patient 1) participating in experiment 1 underwent a nonlinguistic visual search task $[8,20]$. This is an analogous of the visual search task employed in experiment 1 , with the exception that here the high-level shape had no linguistic valence. For a full description of the task see Mangano et al. [20] and Figure 3.

We focused only on the LPpt since the performance of the RPpt and the HC on the same task have been reported in a previous study documenting that lesions to the right parietal cortex impair top-down attentional processes [20]. We used the same procedure as in experiment 1 except that each patient performed 2 practice trials and 18 test trials per stimulus type (A and $B)$.

FIGURE 3

\subsubsection{Data analysis}

For each stimulus type, we calculated accuracy (proportion of correct button presses) and the averaged RTs of the correct button presses. 
In experiment 1, two main statistical analyses were conducted: 1) a within subject analysis comparing the accuracy and the averaged RTs in target-in- $\mathrm{N}$ and in targetin-reversed-N types within each group of participants and 2) a between subject analysis comparing the accuracy and the averaged RTs in target-in-N and in targetin-reversed-N types between the four groups of participants: LPpt; RPpt; HC pref. hand and $\mathrm{HC}$ non-pref. hand.

In experiment 2 we carried out the within subject analysis comparing the accuracy and the averaged RTs in target-in- $\mathrm{N}$ and in target-in-reversed-N types within the left parietal group.

Since sample distributions were strongly skewed (Shapiro-Wilk normality test: targetin-N Accuracy: $W=.61, p<.001$; target-in-reversed-N Accuracy: $W=.81, p<.01$; targetin-N RT: $W=.86, \quad p<.001$; target-in-reversed-N RT: $W=.88, p<.01)$, data were analyzed using non-parametric bias-corrected and accelerated Bootstrapping. We reported $95 \%$ confidence interval of mean difference computed using 10000 replicates, Cohen's d (index of effect size) and the $p$ value.

We performed the robust McCulloch paired test in order to test differences in variability between target-in- $\mathrm{N}$ and target-in-reversed- $\mathrm{N}$ stimuli in each group of participants. Since $7 / 12$ patients suffered from visual field deficit (table 1 ), we also compared the accuracy and the averaged RTs for each stimulus type (target-in-N and target-in-reversed-N) between the two visual hemifields (see Appendix A).

We used $R$ version 3.5.1 for all analyses [21]. A value of $p<.05$ was considered as significant.

\section{Results}

3.1 Experiment 1. Visual search task with linguistic stimuli. 


\subsubsection{Accuracy}

The median and the interquartile range accuracies for the target-in- $\mathrm{N}$ and target-inreversed-N stimuli, respectively, were: $98(I Q R=4.12)$ and $94.25(I Q R=7.92)$ for the HC pref. hand group; $100(I Q R=7.00)$ and $92(I Q R=4.00)$ for the HC non-pref. hand group; $100(I Q R=6.08)$ and $96.15(I Q R=6.17)$ for the $L P p t ; 100(I Q R=.00)$ and 100(IQR=7.69) for the RPpt.

There were no significant differences between the stimulus types in each group ( $\mathrm{HC}$ pref. hand: $M_{d}=2.99,95 \% \mathrm{Cl}:-.38,7.05, d=.66, p=.11 ; \mathrm{HC}$ non-pref. hand: $M_{d}=-.05$, 95\%Cl:-10.14, 4.00, $d=.00, p=1$; LPpt: $M_{d}=2.83,95 \% \mathrm{Cl}:-1.90,8.62, d=.36, p=.31$; RPpt: $\left.M_{d}=.11,95 \% \mathrm{Cl}:-4.23,3.20, d=.02, p=.75\right)$. Robust McCulloch paired test showed a significant difference between the variability of target-in- $\mathrm{N}$ and target-inreversed-N stimuli in $\mathrm{HC}$ pref. hand group $(S=280.86, p<.05)$ but not in the other three groups (HC non-pref. hand: $S=93.17, p=.21$; LPpt: $S=79.42, p=.35$; RPpt: $S=15, p=.68)$.

The between subject analysis revealed that for each stimulus type, there was no significant difference between the accuracies of the four groups of participants (Table 3).

FIGURE 4

TABLE 3

\subsubsection{Averaged RTs}

The within subject analysis showed that the RTs of the HC pref. hand $\left(M_{d}=1474.91\right.$, $95 \% \mathrm{Cl}: 22.01,2631.33, d=.68, p<.05)$, the $\mathrm{HC}$ non-pref. hand $\left(M_{d}=934.39\right.$, $95 \% \mathrm{Cl}: 380.98,1698.21, d=.82, p<.01)$ and the RPpt $\left(M_{d}=2672.13,95 \% \mathrm{Cl}: 1366.55\right.$, 
$3946.49 ; d=1.58, p<.001)$ were significantly longer for target-in- $N$ than for target-inreversed-N stimuli (Figure 5). In contrast, RTs for the two stimulus types were not significantly different in LPpt $\left(M_{d}=699.92,95 \% \mathrm{Cl}:-367.79,1691.21, d=.46, p=.20\right)$.

FIGURE 5

FIGURE 6

Robust McCulloch paired test showed no significant difference between the variability of target-in- $\mathrm{N}$ and target-in-reversed-N stimuli in each group (HC pref. hand: $S=154, p=.86$; HC non-pref. hand: $S=106, p=.31$; LPpt: $S=56, p=1.00$; RPpt: $S=2, p=.08)$.

Two participants (i.e., patient 4 and one $\mathrm{HC}$ responding with her non-preferred hand) showed low levels (<80\%) of accuracy (figure 4). Re-analysis of the within comparison removing the data of these two participants showed again that the left parietal patient group was the only group with no significant difference between target-in- $\mathrm{N}$ and target-in-reversed-N stimuli ( $\mathrm{HC}$ pref. hand: $M_{d}=1474.91$, $95 \% \mathrm{Cl}: 22.01, \quad 2631.33, \quad d=.68, \quad p<.05 ; \quad H C$ non-pref. hand: $M_{d}=1095.45$, 95\%Cl:557.48, 1936.62, $\quad d=1.02, \quad p<.01 ; \quad \mathrm{RPpt}: \quad M_{d}=2672.13, \quad 95 \% \mathrm{Cl}: 1366.55$, 3946.49; $d=1.58, p<.001$; LPpt: $\left.M_{d}=674.31,95 \% C l:-535.96,1893.72 ; d=.41, p=.27\right)$. The between subject analysis revealed that for each stimulus type, there were no other significant differences between the RTs of the four groups of participants (Table 4).

TABLE 4

\subsection{Experiment 2. Visual search task with non-linguistic stimuli.}

\subsubsection{Accuracy}


The median and the interquartile range accuracies for stimulus type $A$ and stimulus type B, respectively, were: $75(I Q R=23.44)$ and $97.22(I Q R=6.08)$.

The patients were significantly less accurate in stimulus type $A$ than in stimulus type B $\left(M_{d}=-21.268,95 \% \mathrm{Cl}:-33.75,-8.33 ; d=-1.21 ; p<.01\right)$.

Robust McCulloch paired test showed a significant difference between the variability of stimulus type $A$ and stimulus type $B(S=52, p<.0001)$.

\subsubsection{Averaged RTs}

The median and the interquartile range RTs for stimulus types $A$ and $B$, respectively, were: $34291 \mathrm{~ms}(I Q R=20552 \mathrm{~ms})$ and $10799.65 \mathrm{~ms}(I Q R=25485.17 \mathrm{~ms})$. The patients showed significantly longer RTs for stimulus type A than B $\left(M_{d}=16230.51\right.$, 95\%Cl:4916.996, 25916.96; $d=1.10 ; p<.01)$.

Robust McCulloch paired test showed no significant difference between the variability of stimulus type $A$ and stimulus type $B(S=26, p=.66)$.

\section{Discussion}

The present study investigated the performance of patients with focal, unilateral parietal lesion and $\mathrm{HC}$ in a unique feature visual search task with letters and reversed letters stimuli. HC presented with significantly longer RTs when the target bar was embedded in ' $N$ ' among its mirror reversed ' $U$ ', confirming the visual search asymmetries known as the "reversed letter effect" $[1,6]$. We found this reversed letter effect also in right but not in left parietal patients.

Such hemispheric specialization within the parietal cortex is in line with a previous study showing that rTMS on the left PPC facilitated visual search for a letter among its mirror images [7]. Therefore, left (but not right) PPC rTMS and left (but not right) 
lesions involving PPC had a specific impact on the reversed letter effect, i.e. they slow down the access to the top-down letter recognition and 'paradoxically' reduce the RTs on finding ' $N$ ' among reversed-N. In contrast, our LPpt were significantly slower and less accurate when the visual search task required to find an oblique target bar embedded in non-linguistic stimuli. This result reveals that LPpt present the top-down object recognition interfering with bottom up feature detection only when the salient stimulus is embedded in an object shape without any linguistic valence.

These findings double dissociate with those of previous studies using the same nonlinguistic visual search. In these cases, both rTMS [8] and brain lesions [20] facilitated performance when involving the right, but not left, PPC.

These modality-specific effects support the idea of a hemispheric specialization of attentional processes within the parietal cortex, according to whether the information involves or not a linguistic association.

The literature on neurological patients reports more severe attentional deficits following right as compared with left hemispheric lesions [22]. An asymmetry in the hemispheric control of attention with a dominance of the right hemisphere either for spatial and non-spatial control has been reported [22-26].

A somewhat different account comes from other neuropsychological and rTMS studies that have emphasized different roles for the right and left hemisphere according to the ability to biasing selection to and from salient stimuli, respectively. Mevorach et al. reported that patients with left PPC damage could attend to both the local and global aspects of a shape, but had difficulty selecting whichever aspect was low in salience and ignoring the other, more salient property [27]. 
Our results can be best accounted for taking into consideration the linguistic material of the task-irrelevant information. We show that damage to the left rather than right parietal cortex can have a larger impact on the attentional network when a visual search task involves linguistic stimuli, such as letters. This result would suggest that the visual search task engages brain areas with functional specialization for linguistic attributes in the left hemisphere. If these areas are lesioned, the linguistic attribute does not contribute additionally to interfere with the task, thereby eliminating the reversed letter effect.

This interpretation may be related with the presence of language deficit in our left parietal group. Indeed 4/7 LPpt presented aphasia. Even though our task does not explicitly require any letter or shape recognition, task irrelevant letter recognition interferes with low-level bottom-up salient feature detection involving V1. We suggest that lesions to the left parietal cortex could have impaired both the access to the letter recognition or the attentive manipulation of the linguistic stimulus, thus reducing the top-down interference to the task. This is consistent with the clinical studies reporting that patients with left posterior lesions showed selective impairment in processing written language [28] and that patients with attentional dyslexia following left parietal damage have difficulties in identifying the constituent letters of words they can read [29]. Indeed, our LPpt showed spared reading ability; they could explicitly recognize letters and correctly discriminate among their mirror-reversal. This is in line with some cases of global alexia documenting that patients can still recognize the letter shape and canonical orientation of letters in paper and pencil tasks measuring accuracy indicating intact access to the representation of letters [30-31]. Measuring RTs, we found a lack of interference exerted by the letter in a 
canonical view. This finding suggests that access to shape recognition, although preserved, becomes slower in LPpt.

There are a number of limitations in our study. The number of patients we investigated is rather limited, particularly for the right parietal group. However, the reversed-letter effect was assessed in each group mainly by the difference between the RTs for the two stimulus types. Hence, this assessment is insensitive to any difference in the overall RTs, which may differ between subject groups.

Another potential limit is that we grouped together patients with different neurological aetiologies (i.e., stroke and tumours). A recent contribution to this debate comes from a study [32], showing that there are no significant differences on neuropsychological performance between frontal patients of different aetiologies. It is reasonable to suppose similar conclusion for different lesion locations, included the parietal cortex.

\section{Conclusion}

The present results support the evidence for hemispheric specialization of attentional processes and would stimulate the use of more suitable instruments to assess the subtle alteration of attention after left parietal lesions.

\section{Acknowledgements}

G.R. Mangano's was supported by University of Palermo Grant, L. Zhaoping by the Gatsby Charitable Foundation Grant, L. Cipolotti by a Department of Health's National Institute for Health Research Biomedical Research Centre's funding scheme, M. Bracco by AIRAIzhOnlus-COOP Italia and M. Oliveri by a grant of Italian Ministry of Health(RF-2010-2311282). 
We are grateful to Prof. E. Ladavas and Dr. D. Braghittoni who kindly helped us in collecting clinical data.

\section{Role of the funding source}

These funding institutions did not have any role in the collecting, analysis and interpretation of data.

Declarations of interest: none

\section{References:}

[1] U. Frith, A curious effect of reversed letters explained by a theory of schema, Percept. Psychophys. 16 (1974) 113-116.

[2] A. Treisman, J. Souther, Search asymmetry: A diagnostic for preattentive processing of separable features, J. Exp. Psychol. Gen. 114 (1985) 285-331.

[3] P. Malinowski, R. Hübner, The effect of familiarity on visual-search performance: Evidence for learned basic features, Percept. Psychophys. 63 (2001) 458-463.

[4] J. Shen, E.M. Reingold, Visual search asymmetry: the influence of stimulus familiarity and low-level features, Percept. Psychophys. 63 (2001) 464-475.

[5] J.M. Wolfe, Asymmetries in visual search: an introduction, Percept. Psychophys. $63(2001) 381-389$.

[6] L. Zhaoping, U. Frith, A clash of bottom-up and top-down processes in visual search: The reversed letter effect revisited, J. Exp. Psychol. Hum. Percept. Perform. 37 (2011) 997-1006. 
[7] G.R. Mangano, M. Oliveri, P. Turriziani, D. Smirni, L. Zhaoping, L.Cipolotti, Repetitive transcranial magnetic stimulation over the left parietal cortex facilitates visual search for a letter among its mirror images, Neuropsychologia 70 (2015) 196205.

[8] M. Oliveri, L. Zhaoping, G.R Mangano, P. Turriziani, D. Smirni, L. Cipolotti, Facilitation of bottom-up feature detection following rTMS-interference of the right parietal cortex, Neuropsychologia 48 (2010) 1003-1010.

[9] B. Wilson, J. Cockburn, P.W. Halligan, The behavioural inattention test, Thames Valley Test Company, Bury St. Edmunds, UK,1987.

[10] G. Miceli, C. Laudanna, C. Burani, R. Capasso, Batteria per l'analisi dei deficit afasici, BADA,CEPSAG, Roma, 1994.

[11] A. Capasso, G. Miceli, Esame Neuropsicologico per l'Afasia: E.N.P.A, SpringerVerlag, Milano, 2001.

[12] C. Rorden MRIcro. http://www.cabiatl.com/mricro.

[13] R.C. Oldfield, The assessment and analysis of handedness: The Edinburgh Inventory,Neuropsychologia 9 (1971) 97-114.

[14] C. Barletta-Rodolfi, F.Gasparini, E. Ghidoni, Kit del Neuropsicologo Italiano, Società Italiana di Neuropsicologia, Bologna, 2011.

[15] J.C. Raven, Colored progressive matrices, Lewis, London,1956.

[16] G.A. Carlesimo, C. Caltagirone, G. Gainotti, The mental deterioration battery: Normative data, diagnostic reliability and qualitative analyses of cognitive impairment. The group for the standardization of the mental deterioration battery, Eur. Neurol. 36 (1996) 378-384. 
[17] G.A. Carlesimo, I. Buccione, L. Fadda, A. Graceffa, M. Mauri, S. Lorusso, C. Caltagirone, Standardizzazione di due test di memoria per uso clinico: Breve racconto e Figura di Rey, Nuova Rivista di Neurologia 12 (2002) 3-13.

[18] J.R. Stroop, Studies of interference in serial verbal reactions, J. Exp. Psychol. 18 (1935) 643-662.

[19] J. Cohen, B. MacWhinney, M. Flatt, J. Provost, PsyScope: A new graphic interactive environment for designing psychology experiments, Behav. Res. Meth. Instrum. Comput. 25 (1993) 257-271.

[20] G.R. Mangano, M. Oliveri, P. Turriziani, D. Smirni, L. Zhaoping, L. Cipolotti, Impairments in top down attentional processes in right parietal patients: paradoxical functional facilitation in visual search, Vision Res. 97 (2014) 74-82.

[21] R Core Team, R: A Language and Environment for Statistical Computing, $R$ Foundation for Statistical Computing, Vienna, 2018. Retrieved from https://www.Rproject.org/.

[22] G. Vallar, Spatial neglect, Balint-Homes' and Gerstmann's syndrome, and other spatial disorders, CNS Spectrums 12 (2007) 527-536.

[23] M. Kinsbourne, Hemi-neglect and hemisphere rivalry, Adv. Neurol. 18 (1977) 41-49.

[24] K.M. Heilman, T.V.D. Abell, Right hemisphere dominance for attention, Neurology 30 (1980) 327.

[25] M. Husain, C. Rorden, Non-spatially lateralized mechanisms in hemispatial neglect, Nat. Rev. Neurosci. 4 (2003) 26-36.

[26] M. Corbetta, G.L. Shulman, Spatial neglect and attention networks, Annu. Rev. Neurosci. 34 (2011) 569-599. 
[27] C. Mevorach, G.W. Humphreys, L. Shalev, Effects of saliency, not global dominance, in patients with left parietal damage, Neuropsychologia 44 (2006) 307319.

[28] J.F. Démonet, G.Thierry, D. Cardebat, Renewal of the neurophysiology of language: functional neuroimaging, Physiol. Rev. 85 (2005) 49-95.

[29] L. Shalev, C. Mevorach, G.W. Humphreys, Letter position coding in attentional dyslexia, Neuropsychologia 46 (2008) 2145-2151.

[30] M. Miozzo, A. Caramazza, Varieties of pure alexia: the case of failure to access graphemic representations, Cogn.Neuropsychol.15 (1998) 203-238.

[31] C. Volpato, G. Bencini, F. Meneghello, L.Piron, C. Semenza, Covert reading of letters in a case of global alexia, Brain Lang. 120 (2012) 217-225.

[32] L. Cipolotti, C. Healy, E. Chan, F. Bolsover, F. Lecce, M. White, B. Spanò, T. Shallice, M. Bozzali, The impact of different aetiologies on the cognitive performance of frontal patients, Neuropsychologia 68 (2015) 21-30. 
Table 1. Patients' demographic and clinical characteristics

\begin{tabular}{|c|c|c|c|c|c|c|c|c|c|c|}
\hline Patients & Gender & $\begin{array}{l}\text { Age } \\
\text { (years) }\end{array}$ & $\begin{array}{l}\text { Education } \\
\text { (years) }\end{array}$ & Etiology & Lesion location & $\begin{array}{l}\text { Time } \\
\text { between } \\
\text { lesion and } \\
\text { test }\end{array}$ & Motor deficit & Hemianopsia & $\begin{array}{l}\text { Neglect } \\
\text { (BIT) }\end{array}$ & $\begin{array}{l}\text { Aphasia } \\
\text { (BADA/ENPA) }\end{array}$ \\
\hline Pt1 & $\mathrm{F}$ & 57 & 17 & ischaemic stroke & $\begin{array}{l}\text { L Fron/Par } \\
\text { cortical-subcortical }\end{array}$ & 3 months & $\mathrm{R}$ hemiparesis & absent & absent & global aphasia \\
\hline Pt2 & M & 21 & 12 & pleomorphic xanthoastrocytoma & L Par & 15 months & absent & absent & absent & absent \\
\hline Pt3 & $\mathrm{F}$ & 52 & 23 & haemorrhagic stroke & LTemp, Par/Occ & 60 months & absent & $\mathrm{R}$ hemianopsia & absent & fluent aphasia \\
\hline Pt4 & $\mathrm{F}$ & 47 & 13 & meningioma & L Par & 10 months & absent & $\mathrm{R}$ hemianopsia & absent & absent \\
\hline Pt5 & M & 67 & 13 & haemorrhagic stroke & L Temp/Par & 27 months & $\begin{array}{l}\text { impairment of } \\
\text { deambulation }\end{array}$ & $\mathrm{R}$ hemianopsia & absent & fluent aphasia \\
\hline Pt6 & $\mathrm{F}$ & 44 & 11 & anaplastic xanthoastrocytoma & LTemp/Par & 6 months & $\begin{array}{l}\mathrm{R} \text { arm } \\
\text { incoordination }\end{array}$ & $\mathrm{R}$ hemianopsia & absent & absent \\
\hline Pt7 & M & 50 & 13 & haemorrhagic stroke & L Fron, Bas G,Temp/Par/Occ & 84 months & $\mathrm{R}$ hemiplegia & diplopia & absent & non-fluent aphasia \\
\hline Pt8 & M & 46 & 8 & meningioma & R Par & 18 months & absent & absent & absent & absent \\
\hline Pt9 & $\mathrm{F}$ & 59 & 17 & meningioma & $\mathrm{R}$ Par/Occ & 20 months & $\mathrm{R}$ arm tremor & absent & absent & absent \\
\hline Pt11 & M & 63 & 13 & ischaemic stroke & R Fron/Par/Temp, Bas G & 17 months & L hemiplegia & L hemianopsia & absent & absent \\
\hline Pt12 & $\mathrm{F}$ & 62 & 13 & meningioma & R Temp/Par/Fron & 17 months & absent & L hemianopsia & absent & absent \\
\hline
\end{tabular}

Legend:

$\mathrm{Pt}=$ patient; $\mathrm{M}=$ male; F=female; R=right; L=left; Par=parietal cortex; Temp=temporal cortex; Fron=frontal cortex; Bas G=basal ganglia;

Occ=occipital cortex. 
Table 2.Neuropsychological tests scores

Patients

\begin{tabular}{|c|c|c|c|c|}
\hline Cognitive domain & Task performed & $\begin{array}{c}\begin{array}{c}\text { Left } \\
\text { Parietal } \\
(\mathrm{N}=7)\end{array} \\
\text { mean (SD) }\end{array}$ & $\begin{array}{c}\begin{array}{c}\text { Right } \\
\text { Parietal } \\
(\mathrm{N}=5)\end{array} \\
\text { mean (SD) }\end{array}$ & p-value \\
\hline General intellectual functioning & $\mathrm{CRPM}^{\S}$ (range $0-36$; cut off $\geq 18,96$ ) & $26.1(5.6)$ & $29.9(7.3)$ & .43 \\
\hline Word retrieval & Object $^{\S}$ (range $0-10$; cut off $\leq 8.2$ ) & $8(2.6)$ & $10(0)$ & .12 \\
\hline Verbal Fluency & Phonemic $^{\S}$ (cut off $\geq 17,35$ ) & 28(13.7) & $35.7(3.7)$ & .26 \\
\hline Verbal memory & Rey's word list delayed recall ${ }^{\S}$ (range $0-15$; cut off $\geq 4,69$ ) & $7.6(0.1)$ & $10.3(3.1)$ & .31 \\
\hline Non verbal memory & Rey's figure-b delayed recall ${ }^{\S}$ (range $0-36$; cut off $\geq 6,33$ ) & $13.1(2.7)$ & $19(2.7)$ & $.03^{*}$ \\
\hline Executive functions & Stroop $\mathrm{C} / \mathrm{W}^{\dagger}$ (cut off=36.92) & 13.4(1.8) & $15.7(4.5)$ & .46 \\
\hline Visual perceptual functions & Conventional Subtests (BIT), raw scores (range 0-146; cut off 129) & $142.7(2.3)$ & $142.5(5.7)$ & .93 \\
\hline
\end{tabular}

Legend:

${ }^{*} \mathrm{p}$-value $<.05$, two tailed t-test;

$\S=$ scores are age and education corrected

${ }^{\dagger}=$ Reaction Times in sec.;

CRPM, Colored Raven's Progressive Matrices;

Stroop C/W, StroopColor/Word interference; BIT, Behavioural Inattention Test. 
Table 3.Experiment 1. Between subject analysis comparing the accuracy in target-in$\mathrm{N}$ and in target-in-reversed-N types between the four groups of participants.

\begin{tabular}{|c|c|c|}
\hline & Target-in- $N$ & Target-in-reversed-N \\
\hline LPpt vs. HC pref. hand & $\begin{array}{l}M_{d}=-.38,95 \% C l:-3.71,2.51, d= \\
-.12, p=.79\end{array}$ & $\begin{array}{l}M_{d}=-.19, \quad 95 \% C l:-8.91, \quad 5.19 \\
d=-.03, p=.95\end{array}$ \\
\hline $\begin{array}{l}\text { LPpt vs. HC non-pref. } \\
\text { hand }\end{array}$ & $\begin{array}{l}M_{d}=2.68,95 \% C l:-1.97,12.48 \\
d=.32, p=.42\end{array}$ & $\begin{array}{l}M_{d}=-.20,95 \% C l:-9.22,4.50 \\
d=-.02, p=.97\end{array}$ \\
\hline LPpt vs. RPpt & $\begin{array}{l}M_{d}=.21,95 \% \mathrm{Cl}:-4.17,8.09, d= \\
.03, p=.93\end{array}$ & $\begin{array}{l}M_{d}=-2.49, \quad 95 \% \mathrm{Cl}: \\
2.61, d=-.35, p=.48\end{array}$ \\
\hline RPpt vs. HC pref. hand & $\begin{array}{l}M_{d}=-.54,95 \% \mathrm{Cl}:-8.43,3.30 \\
d=-.12, p=.79\end{array}$ & $\begin{array}{l}M_{d}=2.25,95 \% \mathrm{Cl}:-2.33,7.24 \\
d=.43, p=.35\end{array}$ \\
\hline $\begin{array}{l}\text { RPpt vs. HC non-pref. } \\
\text { hand }\end{array}$ & $\begin{array}{l}M_{d}=2.51,95 \% \mathrm{Cl}:-4.55,12.39 \\
d=.27, p=.53\end{array}$ & $\begin{array}{l}M_{d}=2.35,95 \% \mathrm{Cl}:-2.08,6.00 \\
d=.64, p=.25\end{array}$ \\
\hline $\begin{array}{l}\text { HC non-pref. hand vs. } \\
\text { HC pref. hand }\end{array}$ & $\begin{array}{l}M_{d}=-3.04,95 \% \mathrm{Cl}:-13.98,1.22 \\
d=-.41, p=.33\end{array}$ & $\begin{array}{l}M_{d}=-.06,95 \% \mathrm{Cl}:-3.60,4.27 \\
d=-.02, p=.93\end{array}$ \\
\hline
\end{tabular}


Table 4. Experiment 1. Between subject analysis comparing the averaged RTs in target-in- $\mathrm{N}$ and in target-in-reversed- $\mathrm{N}$ types between the four groups of participants.

\begin{tabular}{|c|c|c|}
\hline & Target-in-N & Target-in-reversed-N \\
\hline RPpt vs. HC pref. hand & $\begin{array}{l}M_{d}=4908.65, \quad 95 \% \mathrm{Cl}: \quad-2317.85 \\
12658.53, d=.80, p=.21\end{array}$ & $\begin{array}{l}M_{d}=3570.94,95 \% \mathrm{Cl}: \quad-2661.99, \\
10666.63, d=.64, p=.30\end{array}$ \\
\hline $\begin{array}{l}\text { RPpt vs. HC non-pref. } \\
\text { hand }\end{array}$ & $\begin{array}{l}M_{d}=3674.23, \quad 95 \% \mathrm{Cl}:-5301.12 \\
12075.80, d=.46, p=.40\end{array}$ & $\begin{array}{l}M_{\alpha}=2029.39, \quad 95 \% \mathrm{Cl}: \quad-6033.31, \\
8832.81, d=.28, p=.60\end{array}$ \\
\hline LPpt vs. RPpt & $\begin{array}{l}M_{d}=-3540.15,95 \% \mathrm{Cl}:-11883.73 \\
3642.37, d=-.54, p=.39\end{array}$ & $\begin{array}{l}M_{d=-1538.50,} 95 \% \mathrm{Cl}:-8714.31, \\
4643.05, d=-.27, p=.66\end{array}$ \\
\hline LPpt vs. HC pref. hand & $\begin{array}{l}M_{d}=1238.23, \quad 95 \% \mathrm{Cl}: \quad-2170.74 \\
4595.06, d=.34, p=.47\end{array}$ & $\begin{array}{l}M_{d}=2113.04, \quad 95 \% \mathrm{Cl}: \quad-1896.62, \\
5100.67, d=.52, p=.52\end{array}$ \\
\hline $\begin{array}{l}\text { LPpt vs. HC non-pref. } \\
\text { hand }\end{array}$ & $\begin{array}{l}M_{d}=179.54, \quad 95 \% \mathrm{Cl}: \quad-6434.133 \\
4301.557, d=.03, p=.94\end{array}$ & $\begin{array}{l}M_{d}=450.92, \quad 95 \% \mathrm{Cl}:-5847.31, \\
4099.39, d=.08, p=.86\end{array}$ \\
\hline $\begin{array}{l}\text { HC non-pref. hand vs. } \\
\text { HC pref. hand }\end{array}$ & $\begin{array}{l}M_{d}=1124.55, \quad 95 \% \mathrm{Cl}: \quad-2659.42, \\
7578.22, d=.18, p=.68\end{array}$ & $\begin{array}{l}M_{d}=1573.77, \quad 95 \% \mathrm{Cl}: \quad-2304.14, \\
7233.11, d=.29, p=.51\end{array}$ \\
\hline
\end{tabular}

Figure 1. CT/MRI digitalized images of (a) five LPpt (Pt2, Pt3, Pt4, Pt5, Pt6) and (b) three RPpt patients (Pt8, Pt9, Pt10) mapped using MRlcro software. One colour refers to one patient. Dark violet: one ROI (region of interest); red: all ROls. (For interpretation of the references to colour in this figure legend, the reader is referred to the web version of this article).

Figure 2. Experiment 1. Small fraction of the visual-search displays.

a) In target-in-N stimulus, the target bar was in an object shape ' $N$ ' contained familiar verbal information. b) In target-in-reversed- $\mathrm{N}$ stimulus, the target bar was in an object shape ' $U$ ' which was the mirror reversal of ' $N$ '. 
Figure 3. Experiment 2. Small fraction of the visual-search displays.

a) In stimulus type $A$, the $X$-shapes in the search array had identical shape, causing confusion at the top-down object shape recognition level whereas the task was at the orientation feature detection level.

b) In stimulus type $B$, the $X$-shape containing the target bar was thinner than all other X-shapes in the image.

Figure 4. Experiment 1. Scattergram of accuracy with the two stimulus type, targetin- $\mathrm{N}$ and target-in-reversed- $\mathrm{N}$, respectively, on the $\mathrm{X}$ and $\mathrm{Y}$ axes, and points distinguished by group. (For interpretation of the references to colour in this figure legend, the reader is referred to the web version of this article).

Figure 5. Experiment 1. Median RT of healthy controls, LPpt and RPpt' in the visual search task. Error bars represent the interquartile range.

Figure 6. Experiment 1. Scattergram of the averaged RTs with the two stimulus type, target-in- $\mathrm{N}$ and target-in-reversed- $\mathrm{N}$, respectively, on the $\mathrm{X}$ and $\mathrm{Y}$ axes, and points distinguished by group. (For interpretation of the references to colour in this figure legend, the reader is referred to the web version of this article).

Appendix A: Visual hemifields analysis

\section{Experiment 1}

\subsection{Accuracy}

No significant difference was found in each group between the accuracies of target$\mathrm{N}$ stimuli in the right and in the left hemifield (HC pref. hand: $M d=1.46,95 \% \mathrm{Cl}:-2.56$, 
8.40, $d=.16, p=.67$; HC non-pref. hand: $M d=-4.32,95 \% C l:-24.87,2.31, d=-.22$, $p=.52$; LPpt: $M d=-3.70,95 \% \mathrm{Cl}:-7.23,1.10, d=-.56, p=0.07 ; \mathrm{RPpt}: M d=2.56,95 \% \mathrm{Cl}: 0$, 5.24, $d=.45, p=0.26$ ) and target-in-reversed-N stimuli ( $\mathrm{HC}$ pref. hand: $M d=-1.36$, 95\%Cl:-6.08, 2.50; $d=-.19, p=.56$; HC non-pref. hand: $M d=3.05,95 \% \mathrm{Cl}:-1.73,8.33$; $d=.37, p=.23 ;$ LPpt:Md=.14, $95 \% \mathrm{Cl}:-6.21,7.51 ; p=.95 ; d=.02 ;$ RPpt: $M d=6.10$, $95 \% \mathrm{Cl}: 0,12.38 ; d=.73, p=.06)$.

\subsection{Averaged RTs}

In each group of participants there were not significant differences between the averaged RTs in the right and in the left hemifield both for target-in-N stimulus ( $\mathrm{HC}$ pref. hand: $M d=486.17,95 \% \mathrm{Cl}:-657.72,2154.08, d=.22, p=.46$; $\mathrm{HC}$ non-pref. hand: $M d=1024.44,95 \% \mathrm{Cl}:-362.48,5301.97, d=.27, p=.44$; LPpt: $M d=1572.81,95 \% \mathrm{Cl}:-$ 833.34, 6269.29, $d=.34, p=.34$; RPpt: $M d=-2461.28,95 \% C l:-12359.82,1740.49, d=-$ $.31, p=0.49$;) and target-in-reversed-N stimulus (HC pref. hand: $M d=812.71,95 \% \mathrm{Cl}$ :378.19, 2469.65; $d=.35, p=.25 ; \mathrm{HC}$ non-pref. hand: $M d=2399.35,95 \% \mathrm{Cl}:-2657.75$, 9674.88; $d=.66, p=.43 ;$ LPpt: $M d=-20.20,95 \% \mathrm{Cl}:-1622.40,1461.57, d=-.01, p=.98$; RPpt: $M d=1453.02,95 \% \mathrm{Cl}:-2667.23,4887.34, d=.29, p=.46)$.

\section{Experiment 2}

\subsection{Accuracy}

The patients were significantly less accurate in the right than in the left hemifield for stimulus A $(M d=-8.09,95 \% \mathrm{Cl}:-18.52,-2.08 ; d=-.80, p<.05)$ but not for stimulus $\mathrm{B}$ $(M d=-3.57,95 \% \mathrm{Cl}:-12.92,3.70 ; d=-.32, p=.38)$.

\subsection{Averaged RTs}


There were not significant differences between RTs in the right and in the left hemifields both for $\mathrm{A}(\mathrm{Md}=3857.23,95 \% \mathrm{Cl}:-1182.97,16748.19, \mathrm{~d}=.20, \mathrm{p}=.60)$ and $\mathrm{B}$ stimuli (Md=9115.03, 95\%Cl:-1394.40, 38783.35, $d=.38, p=.43)$. 